Dimogerontakis, E. [et al.]. MeshDapp: Blockchain-enabled sustainable business models for networks. A: International Conference on Economics of Grids, Clouds, Systems and Services. "Economics of Grids, Clouds, Systems, and Services, 16th International Conference, GECON 2019: Leeds, UK, September 17-19, 2019: proceedings". Berlín: Springer, 2019, p. 286-290. ISBN

978-3-030-36027-6.

The final authenticated version is available online at https://doi.org/10.1007/978-3-030-36027-6\_25.

\title{
MeshDapp - Blockchain-enabled Sustainable Business Models for Networks
}

\author{
Emmanouil Dimogerontakis ${ }^{1}$, Leandro Navarro ${ }^{1}$, Mennan Selimi ${ }^{1,2}$, \\ Sergio Mosquera ${ }^{1}$, and Felix Freitag ${ }^{1}$ \\ 1 Universitat Politècnica de Catalunya, BarcelonaTech, Spain \\ 2 Max van der Stoel Institute, South East European University, North Macedonia
}

\begin{abstract}
The digital world demands a network infrastructure to supply connectivity to any participant anywhere. Sustainable networks require balanced value flows. Value is connectivity delivered at a material and service cost to compensate, involving diverse participants, ranging from consumers to providers, such as last mile access, regional transport, Internet carriers, or content providers. We focus on the case of wireless mesh networks that deliver connectivity through access points and a mesh network that routes traffic to Internet gateways, provisioned by several device owners and service operators [1-3]. The presented work is motivated by the need for balance and automation among services delivered, costs and incentives for participation in these decentralised networks. This balance is key for achieving extensible network infrastructures that can deliver widespread availability of Internet connectivity with minimal barriers of entry.
\end{abstract}

Keywords: Blockchain, Mesh networks, Network sustainability

\section{Problem statement}

We explore the technological feasibility to implement business and sustainability models that combine retail pricing with wholesale cost, profit distribution and return of investment. A technical enabler is a permissioned blockchain that provides desirable properties such as trusted data feeds (oracles) about traffic and resource consumption, robust and irreversible transaction records (distributed ledger), and inexorable outcomes (smart contracts).

\section{Conceptual Architecture}

A mesh network provides connectivity services such as Internet access, content and local services. We need multiple network devices, such as access points (APs), routers (R), servers (S), and Internet Gateways (GW). Consumers (users) can connect to network services and the Internet through AP devices in various locations, interconnected through several intermediate mesh routers. Servers deliver local services, and one or several gateway nodes are needed to deliver enough Internet connectivity.

Each device $\{A P, R, G W, S\}$ in a mesh has a cost that corresponds to an initial investment on network devices, links, servers (CAPEX) and its maintenance and 
operation (OPEX). This cost can fluctuate according to usage. Similarly to the electricity market, there is need for a market maker, the mediator agent, that finds the optimal retail service prices (e.g. the MBh equivalent to the $\mathrm{kWh}$ ) to balance the demand and supply of connectivity across the different network infrastructure paths.

Such a system can accommodate participation, capacity, growth, variability and sustainability. While the routing protocol tries to optimize the allocation of capacity to traffic, the price optimization by the mediator agent can be used by service providers to compute retail tariffs for traffic and services offered to consumers (price plans). Examples are Internet access when including an Internet GW, or other services when combined with the matching GW/server such as voice calls, content (e.g. radio, TV, video). Each participating network device is rewarded by payments from consumers (who mediates this for later). In addition, these devices can provide connectivity and services to their owners, as both providers and consumers (prosumers).

Services satisfy consumer demand through the effort done by the supply or value chain. In the short term, there must be a balance between the charges in the consumption and the supply side. In the long term, services and the infrastructure should be socially and economically viable, satisfactory to all parts.

The main stakeholders are:

- User: a client of the mesh network. The user has a user interface (web browser or mobile app) and can have different roles (e.g., consumer, provider, network admin).

- Consumer: a user that consumes connectivity in exchange of an economic contribution according to a service contract: a customer of a retailer AP.

- Provider: a user that owns and provides devices $\{A P, R, G W, S\}$ to the infrastructure, or resources to supply connectivity or content services in a given local network expecting a compensation in economic or other terms.

The main components of the model are the following:

- Mesh Network Island: the network devices, the wireless physical connection between them, and the stack of network protocols that enable their connectivity. It is important to clarify that economic incentives are kept separate, not affecting routing and forwarding decisions driven by link quality metrics. Mixing economic with quality choices may lead to routing and forwarding convergence issues.

- Node DB: contains information relevant to the mesh about all the network devices. - Monitoring System: responsible for monitoring the resources being used (i.e. traffic amounts) and providing the necessary data to the rest of the services.

- Mediator: the core agent of the model that, in every mesh island, is responsible for matching the demand and the supply of the offered service as well as maintaining the balances among the different participants. The Mediator applies the logic to compute the distribution of economic value, the settlement to providers in exchange of services, and determines a fair price for retail and aggregate traffic for each usage cycle.

\subsection{Economic Compensation System}

The aim of an economic compensation mechanism is to fairly distribute the economic value of the connectivity supplied by the network among providers: the devices and 
services $\{A P, R, G W, S\}$ which contributed to deliver that value. The amount received from service provision (retail) to consumers has to be distributed across the suppliers, an (approximately) zero-sum balance as in Eq. 1. Retailers (service providers) get funds (monetary units $F_{i}$ ) in exchange to amounts of service provision (Internet traffic or any other service or content) (as byte units $B_{i}$ ):

$$
\begin{aligned}
\text { Collected }(t) & =\sum_{r=1 . . R} \sum_{R=1 \ldots C}\left(B_{r, c}, F_{r, c}\right)=(B, F) \text { in period } p_{t} \\
\text { where }: & r \text { is a retailer among the set } R \\
c & \text { is a customer among the set } C
\end{aligned}
$$

The tuple $(B, F)$ has to (roughly) match the service provided by suppliers in the value chain, considering that the provision of connectivity involves multiple network hops and devices.

The accounting of traffic could be done in detailed retail form per client connection in each device (each client payment is split across the network devices involved in that provision), but we have selected an aggregate form where the sum of all user payments is split among all forwarding reports across all network devices involved in each period. That simplifies the accounting (routers only count bytes forwarded, instead of bytes per each end-client) at the price of small aggregation differences.

$$
\text { Owed }(t)=\sum_{0<f<F} \text { Settle }_{n}(B(t, f), F(t, f)) \text { in period } p_{t}
$$

where: $f$ is a forwarder among the set $F$ $n$ is a settlement event for period $p_{t}$

The service fees collected in a given period can be allocated in settlements proportional to weights in terms of aggregated service (forwarding) units and fee/price units as in Eq. 2.

The goal of the mediator agent is to determine the settlement of owed payments in a mesh island, for each node and time, that optimize Eq. 3 for every user $i$ as consumer (constr. 3b) or provider (device owner), where the settled retribution to each node $($ Owed $)$ satisfies its expressed Price (constr. 3d), and where each device is useful, with positive utility (constr. 3c), such as by satisfying the expected rate of RoI (Return on Investment) combined with its social and economic benefits.

Payments spread across the value/service chain of network devices can be achieved according to the idea of fairness of settlements, such as proportional share [4] or Shapley value in ISPs [5]. To ensure that payments are satisfied in each cycle (constr. $3 \mathrm{~d}$ ) we use a reserve fund which allows us to keep a fund margin across payment cycles. This margin comes from the difference between the customer offered (spot) retail price and the internal aggregate network prices or costs.

$$
\begin{array}{cc}
\underset{c, f, t}{\operatorname{maximize}} & U_{i} \quad(\forall \text { consumer } c \text {,device } f, \text { time } t) \\
\text { subject to } & U(c, t)>0, c=1, \ldots, C, \\
& U(f, t)>0, f=1, \ldots, F, \\
& \text { Owed }(f, t)-\operatorname{Price}(f, t) \geq 0, t=0, \ldots, T
\end{array}
$$


We argue that smart contracts in a blockchain can automatically facilitate, verify and enforce the negotiation or performance of the aforementioned mechanism in a transparent and irreversible way. That can result in value transfers in the form of payments of token units. In the next section we sketch how a blockchain using smart contracts can implement the economic interaction between the components mentioned in the architecture. We materialize our design in the MeshDapp system.

\section{Approach and evaluation}

We have implemented the models in a set of Solidity smart contracts under an Ethereum PoA permissioned and local blockchain ${ }^{3}$, and evaluated its feasibility and operation under a simulated mesh network, as well as integrated in an experimental mesh network. We describe in the poster the overall idea and how the contract architecture has been designed.

\section{Conclusions}

We find the system, smart contract architecture, and the oracle to feed them with monitoring data, to be feasible and able to support the exchange of economic value in exchange of connectivity, between consumers and providers. The resulting decentralized and open model can support the sustainability of the network infrastructure and the services it provides, as well as create opportunities for local participants to generate value, both from the economic and networking perspectives.

\section{Acknowledgement}

This paper has been partially supported by the AmmbrTech Group, the Spanish government TIN2016-77836-C2-2-R and the Catalan government AGAUR SGR 990.

\section{References}

1. Braem, B., Baig Viñas, R., Kaplan, A.L., Neumann, A., Vilata i Balaguer, I., Tatum, B., Matson, M., Blondia, C., Barz, C., Rogge, H., Freitag, F., Navarro, L., Bonicioli, J., Papathanasiou, S., Escrich, P.: A case for research with and on community networks. ACM SIGCOMM Computer Communication Review 43(3) (July 2013) 68-73

2. Baig, R., Roca, R., Freitag, F., Navarro, L.: guifi.net, a crowdsourced network infrastructure held in common. Computer Networks (2015)

3. Kabbinale, A.R., Dimogerontakis, E., Selimi, M., Ali, A., Navarro, L., Sathiaseelan, A., Crowcroft, J.: Blockchain for economically sustainable wireless mesh networks. Concurrency and Computation: Practice and Experience 0(0) e5349 e5349 cpe.5349.

4. Feldman, M., Lai, K., Zhang, L.: The proportional-share allocation market for computational resources. IEEE Trans. Parallel Distrib. Syst. 20(8) (August 2009) 1075-1088

5. Stanojevic, R., Laoutaris, N., Rodriguez, P.: On economic heavy hitters: Shapley value analysis of 95th-percentile pricing. In: Proceedings of the 10th ACM SIGCOMM Conference on Internet Measurement. IMC '10, New York, NY, USA, ACM (2010) 75-80

\footnotetext{
${ }^{3}$ http://dsg.ac.upc.edu/meshdapp
} 